\title{
Rheophysical investigation in concentrated particle suspensions
}

S. Wiederseiner, C. Ancey, N. Andreini \& M. Rentschler

Laboratoire d'Hydraulique Environnementale

Ecole polytechnique fédérale de Lausanne

The Society of Rheology 80th Annual Meeting

August 3 - 8, 2008 - Monterey, California

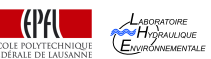

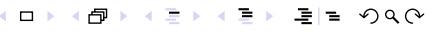


(1) Gravity driven geophysical flows

(2) Rheology and Rheophysics

(3) Concentrated particle suspensions

(4) Measurement techniques and setup

(5) Results

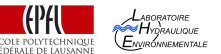

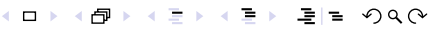




\section{Geophysical flows}

- Snow avalanches

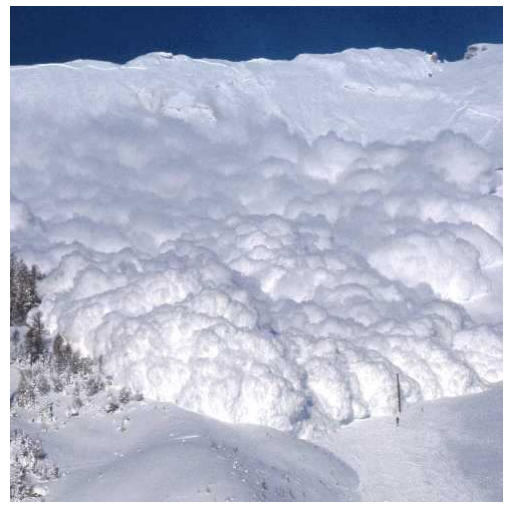

Photo SLF

Labogatolire Higraulouve ECOLE POLLYTECHNIOUJE
FEDERALE DE LAUSANNE EMVIRONNEMENTALE 


\section{Geophysical flows}

\section{- Snow avalanches}

- Mudflows

- Pyroclastic flows

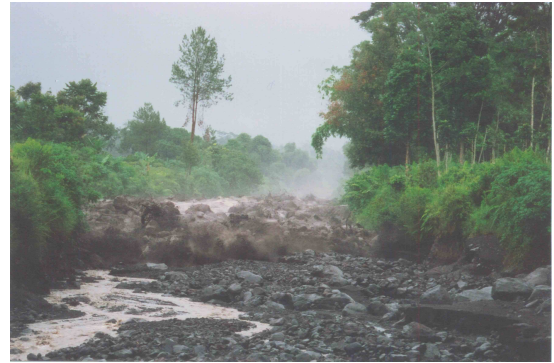

(P)A

Laboratoire
Hugraulave

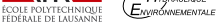

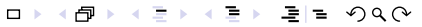




\section{Geophysical flows}

- Snow avalanches

- Mudflows

- Pyroclastic flows

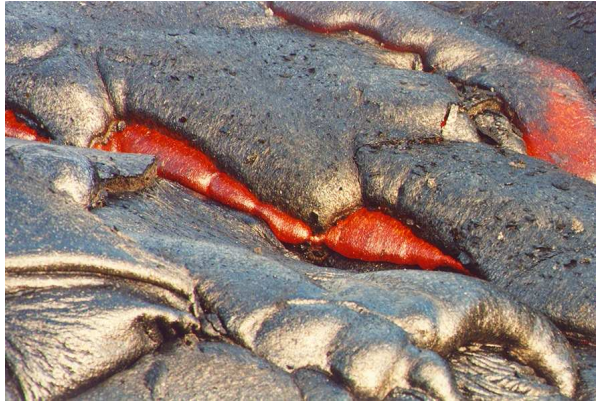

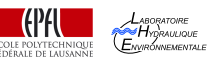

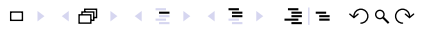




\section{Geophysical flows}

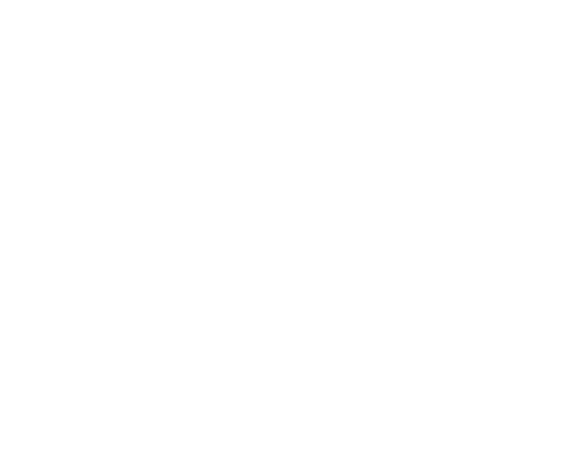




\section{(1) Gravity driven geophysical flows}

\section{(2) Rheology and Rheophysics}

Concentrated particle suspensions

- Properties of the suspensions

- Temperature effects

- Wavelength effects

Measurement techniques and setup

- FPIV / FPTV techniques

- Measurement setup

Results

- Validation

- Velocity profile of concentrated suspensions

- Flow curve derivation 


\section{Geophysical flows}

Complex fluids

- Particles

- Material

- Shape

- Granulometry

- Rugosity

- Interstitial fluids

- Viscosity
How do we measure the rheological properties?

- Yield stress

- Shear-thinning, Shear-thickening

- Thixotropy, rheopecty

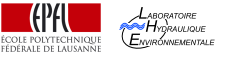




\section{Geophysical flows}

Complex fluids

- Particles

- Material

- Shape

- Granulometry

- Rugosity

- Interstitial fluids

- Viscosity
How do we measure the rheological properties?

- Yield stress

- Shear-thinning, Shear-thickening

- Thixotropy, rheopecty

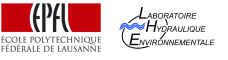




\section{Consequences for the rheologist}

- $\left(\begin{array}{l}\mathrm{T} \\ \Omega\end{array}\right) \rightarrow\left(\begin{array}{l}\tau \\ \dot{\gamma}\end{array}\right)$

(3) Wide gap (because of the granulometry)

$\mathrm{T}:$ Total Torque

$\Omega$ : Angular velocity

$\tau$ : shear stress

$\dot{\gamma}$ : shear rate
Solve the Couette inverse problem

$\Rightarrow$

$$
\begin{aligned}
\tau(r) & =\frac{T}{2 \pi r^{2} h} \\
\Omega & =\int_{R_{\text {in }}}^{R_{\text {out }}} \frac{\dot{\gamma}(r)}{r} d r
\end{aligned}
$$

\footnotetext{
$r \quad:$ Radius

$\mathrm{h} \quad$ : Height of fluid

$R_{\text {in/out }}$ : Radius of the inner/outer cylinder
} 


\section{Consequences for the rheologist}

Example : an artificial Herschel-Bulkley fluid $\tau=\tau_{y}+K \dot{\gamma}^{n}$

$s=\frac{R_{\text {in }}}{R_{\text {out }}}=0.9$

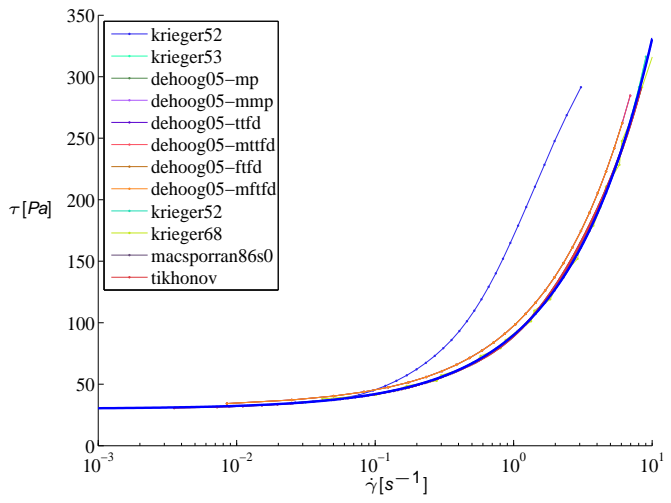

(1) (1) Laboratome

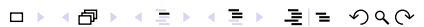




\section{Consequences for the rheologist}

The same fluid with a wide-gap geometry

$s=\frac{R_{\text {in }}}{R_{\text {out }}}=0.2$

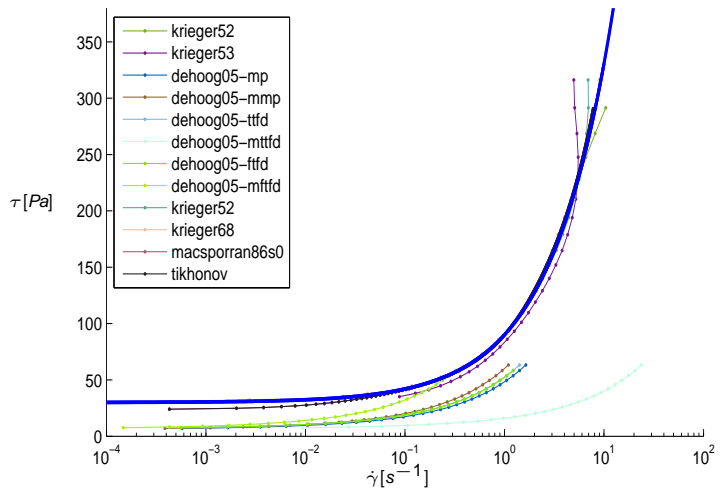

ECOLE F'OLYTECHNIOUJE

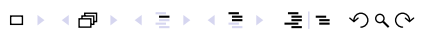




\section{Example : a polymeric gel}

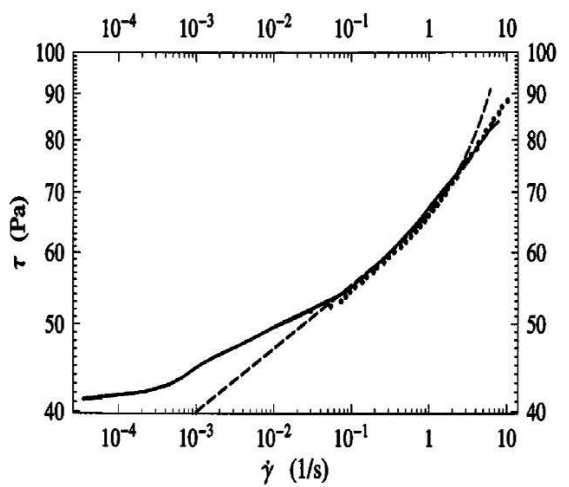

Ancey, J.Rheology 49 (2005) 441-460

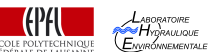




\section{Example : a particle suspensions}

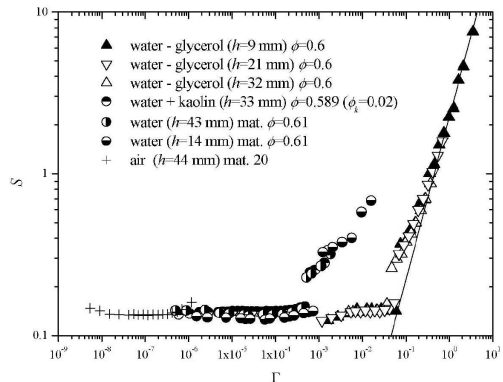

Ancey, J. Rheol. 45 (2001)1421-1439

$S$ : adimensionalized shear stress

$\Gamma$ : adimensionalized angular velocity 


\section{Example : a particle suspensions}

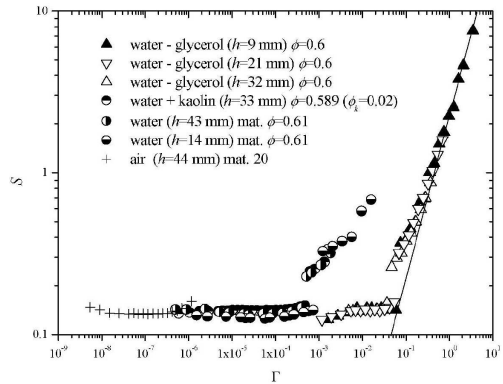

Ancey, J. Rheol. 45 (2001)1421-1439

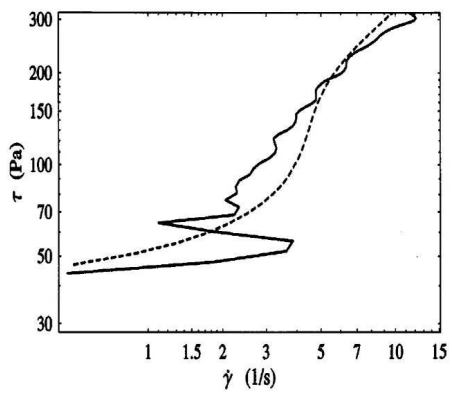

Ancey, J.Rheology 49 (2005) 441-460

$S$ : adimensionalized shear stress

$\Gamma$ : adimensionalized angular velocity

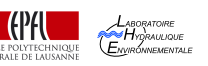


- Shear localization?

- Particle segregation?

- Particle rugosity?

- Particle migration?

- Particle Shape?

- Slipping?

- Ordering?

Do we measure the material property...

...or some flow artifacts?

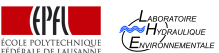

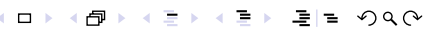




\section{Classical and optical rheometry}

Continuum mechanics approach $\Downarrow$

Classical rheometry

T and $\Omega$

$\Downarrow$

Solve the Couette inverse problem $\Downarrow$ $\tau$ and $\dot{\gamma}$
Rheophysical

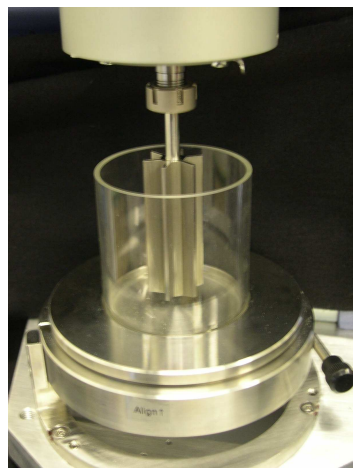
approach

$\Downarrow$

Clear suspensions

$\Downarrow$

Particle motion (FPIV / FPTV) $\Downarrow$

Differentiate the velocity profile $\Downarrow$ $\tau$ and $\dot{\gamma}$

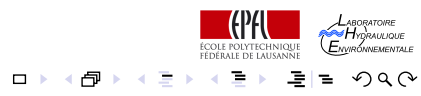




\section{Gravity driven geophysical flows}

\section{Rheology and Rheophysics}

3 Concentrated particle suspensions

- Properties of the suspensions

- Temperature effects

- Wavelength effects

Measurement techniques and setup

- FPIV / FPTV techniques

- Measurement setup

Results

- Validation

- Velocity profile of concentrated suspensions

- Flow curve derivation

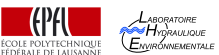


Properties of the suspensions

\section{Where are the properties comming from?}

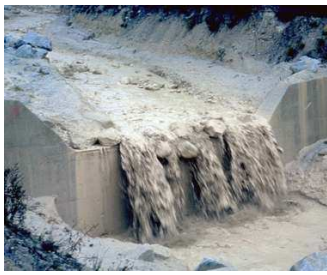

Interesting flows
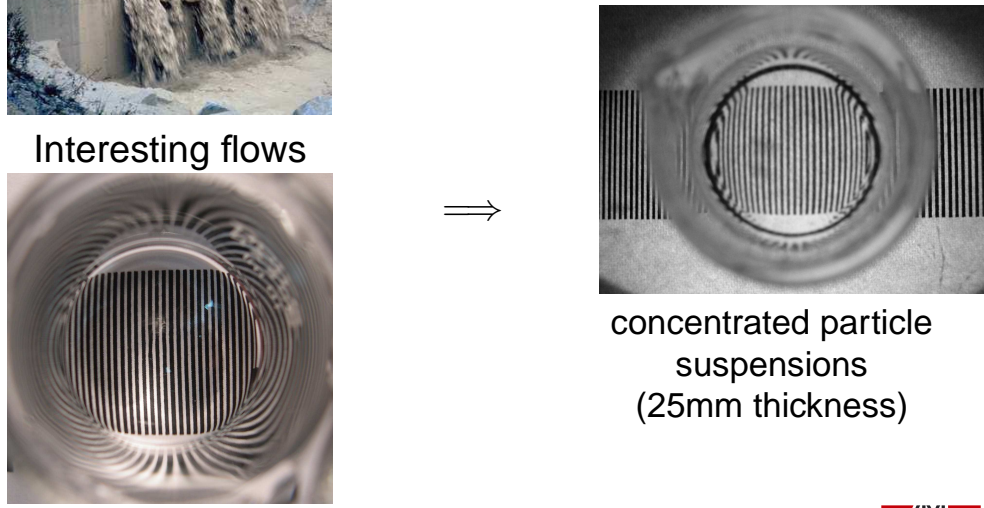

concentrated particle suspensions (25mm thickness)

Optical methods

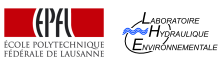

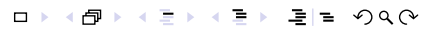




\section{Properties of the suspensions}

\section{The simplest complex fluid}

- Iso-index $\Rightarrow$ transparency

- Iso-density $\Rightarrow$ No gravitation effects

- Molecular tagging of the particles

$\Rightarrow$ the laser excite the fluorescence

Particles

- Shape : spherical

- Granulometry
Fluid

- Three fluids mixture

- Newtonian

- Viscosity : variable 


\section{Temperature effects}

\section{Temperature effects}

- Density
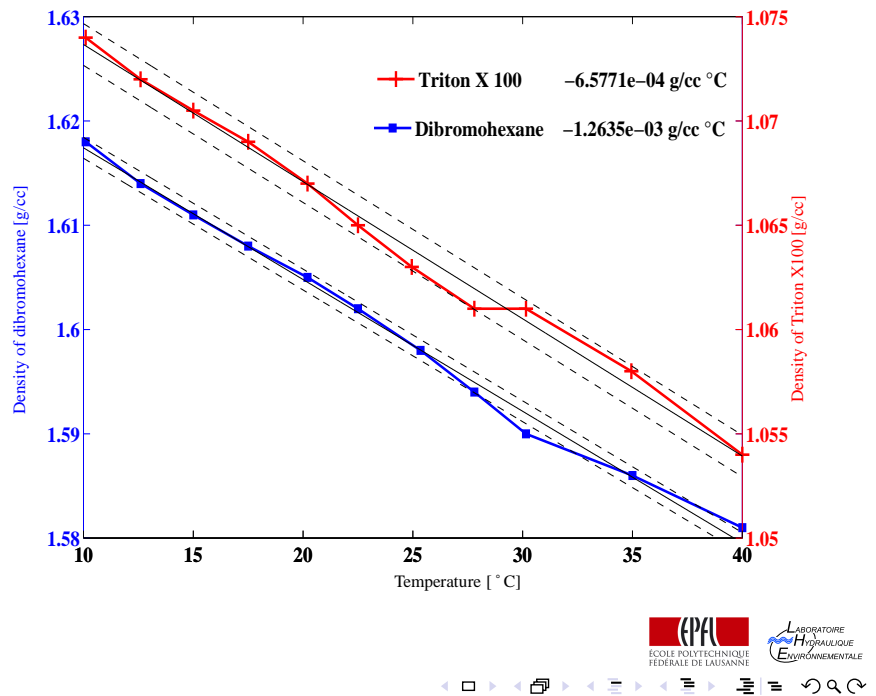


\section{Temperature effects}

\section{Temperature effects}

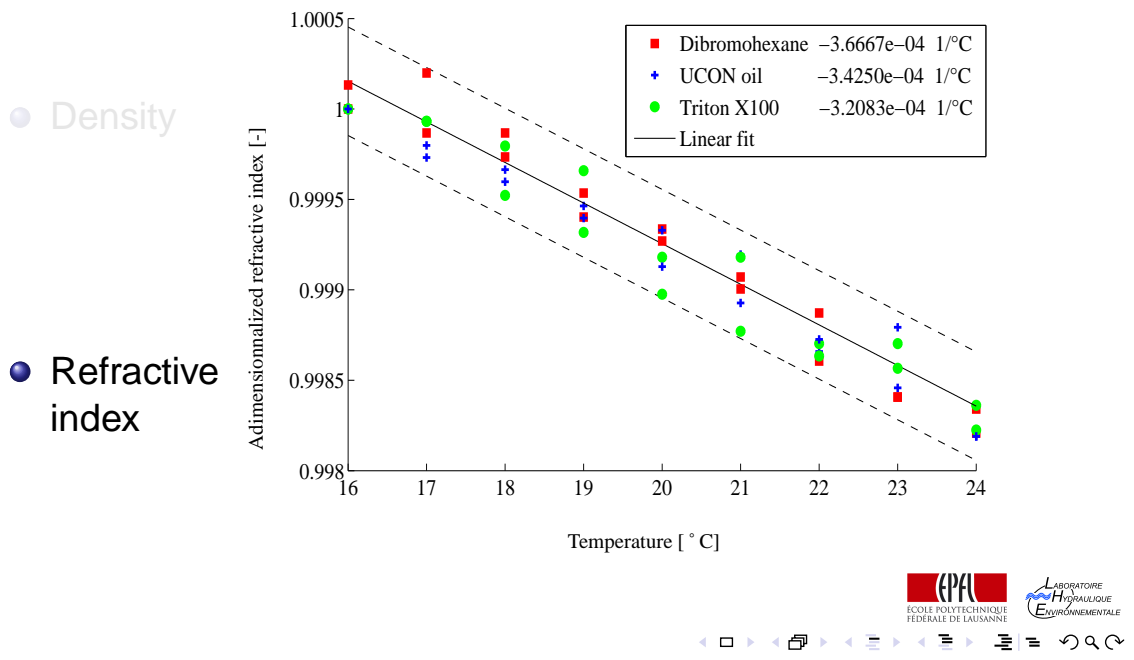




\section{Temperature effects}

\section{Temperature effects on the light transmission}

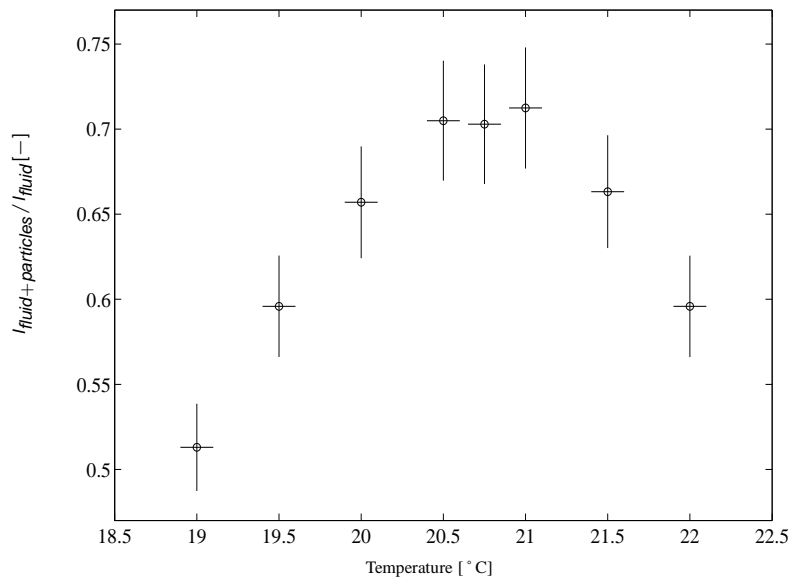

(1)(1 Laboratome ECOLE POLYTECHNIOUJE EMMirónNEMENTALE

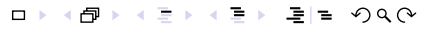


Wavelength effects

Refractive index mismatch effects on the transmission

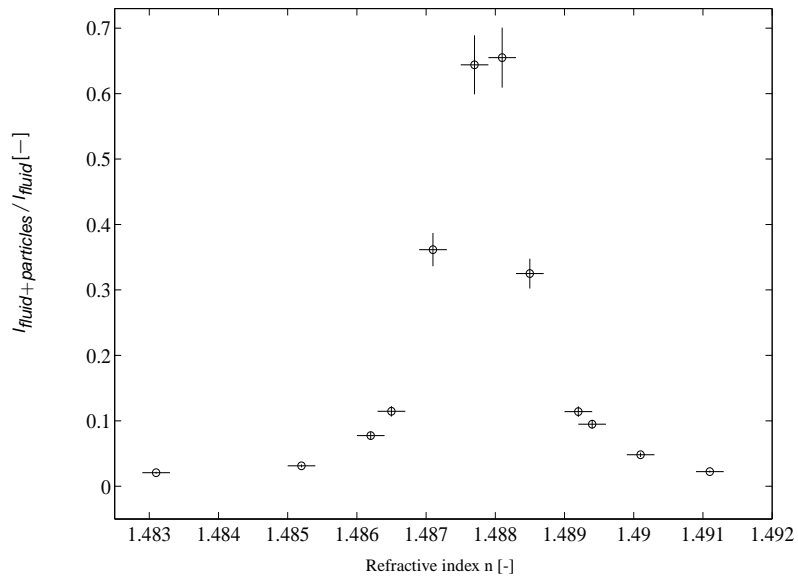

IEPALI

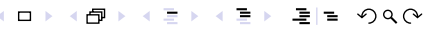




\section{Wavelength effects}

\section{Wavelength effects}
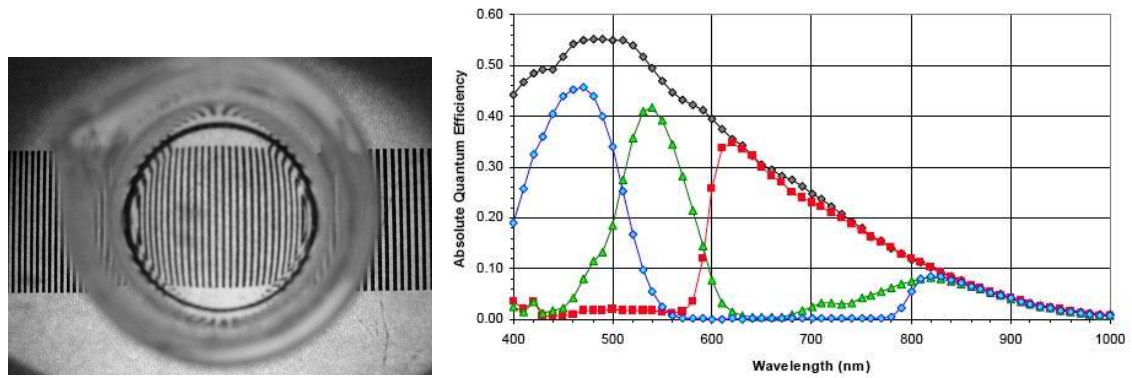

Monochrome with Microlens $\rightarrow-$ Red $\rightarrow$ - Green $\rightarrow-$ Blue

\section{(P) $P$ (1)}

Laboratone

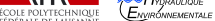

口 吕 
Wavelength effects

Wavelength effects

RGB picture with a color CCD camera :

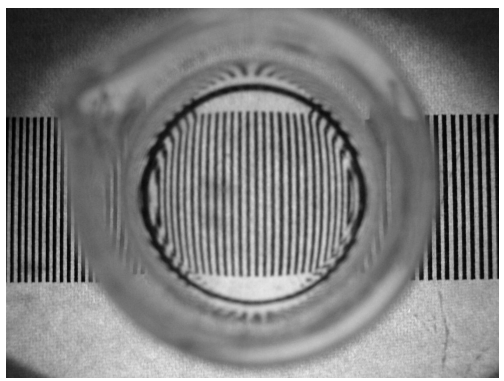

Blue component

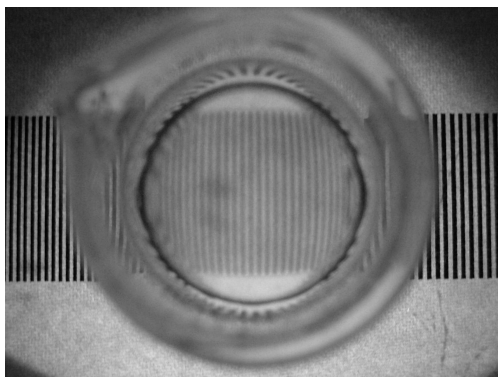

Red component

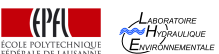

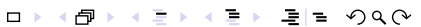




\section{Gravity driven geophysical flows}

\section{Rheology and Rheophysics}

Concentrated particle suspensions

- Properties of the suspensions

- Temperature effects

- Wavelength effects

(4) Measurement techniques and setup

- FPIV / FPTV techniques

- Measurement setup

Results

- Validation

- Velocity profile of concentrated suspensions

- Flow curve derivation

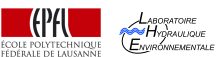


FPIV / FPTV techniques

\section{Measurement methods}

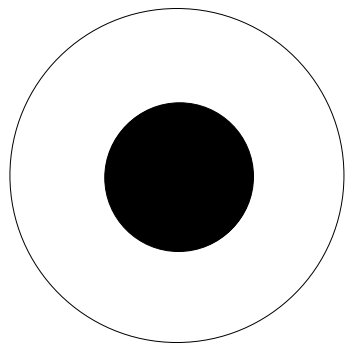


FPIV / FPTV techniques

\section{Measurement methods}

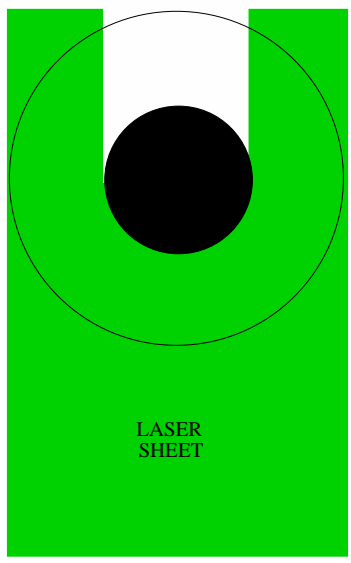


FPIV / FPTV techniques

\section{Measurement methods}

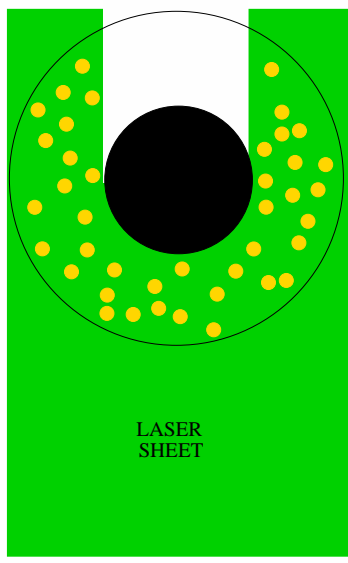

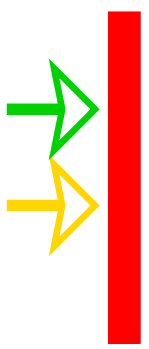

FILTER

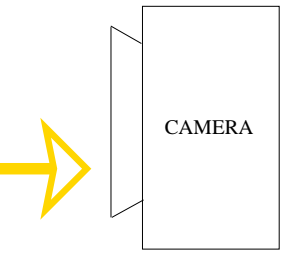




\section{FPIV / FPTV techniques}

\section{Measurement methods}
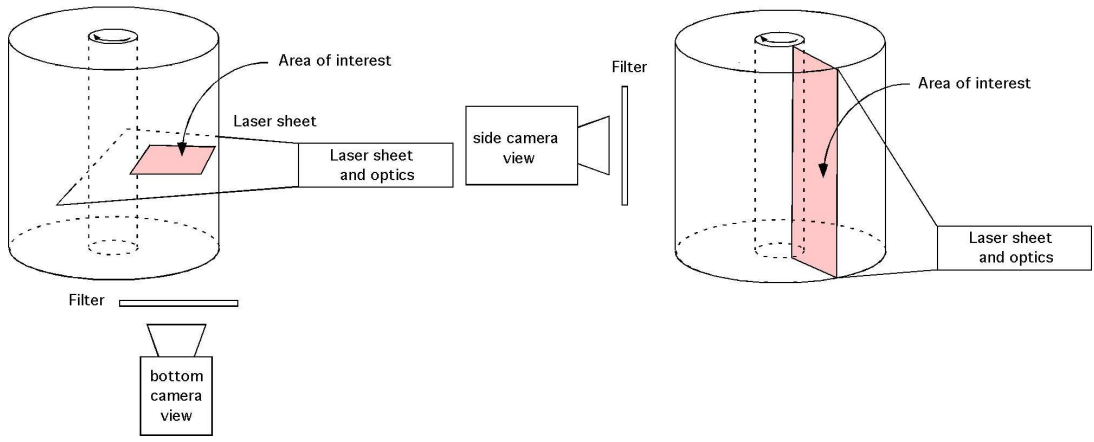


\section{Measurement setup}

\section{The setup}
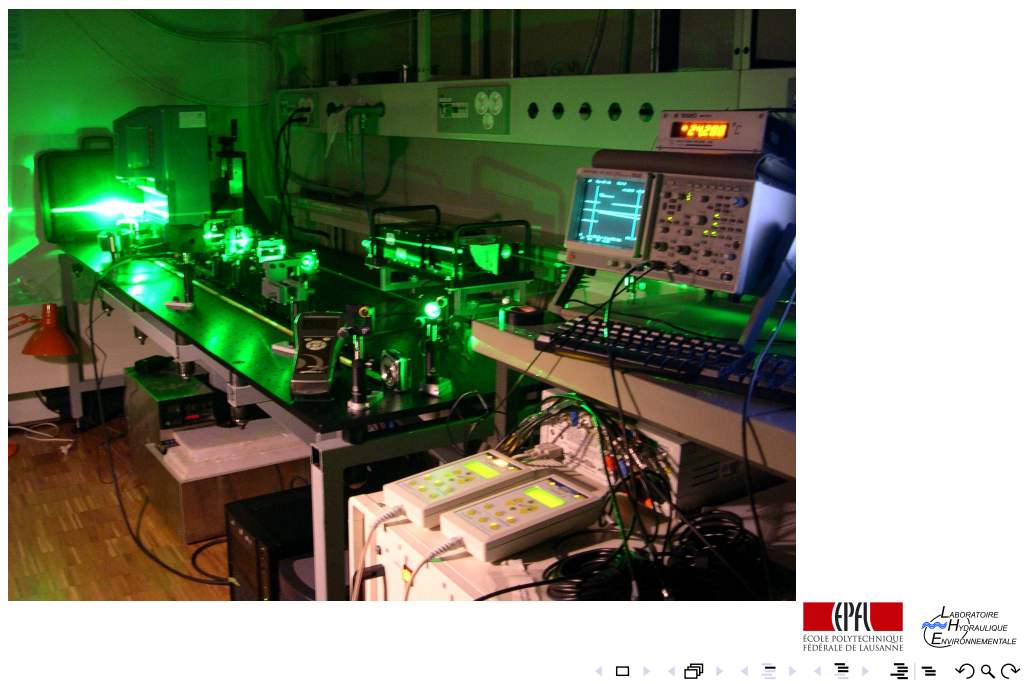


\section{Gravity driven geophysical flows}

\section{Rheology and Rheophysics}

Concentrated particle suspensions

- Properties of the suspensions

- Temperature effects

- Wavelength effects

Measurement techniques and setup

- FPIV / FPTV techniques

- Measurement setup

5 Results

- Validation

- Velocity profile of concentrated suspensions

- Flow curve derivation

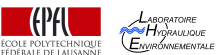

口 吕 


\section{FPIV Images}
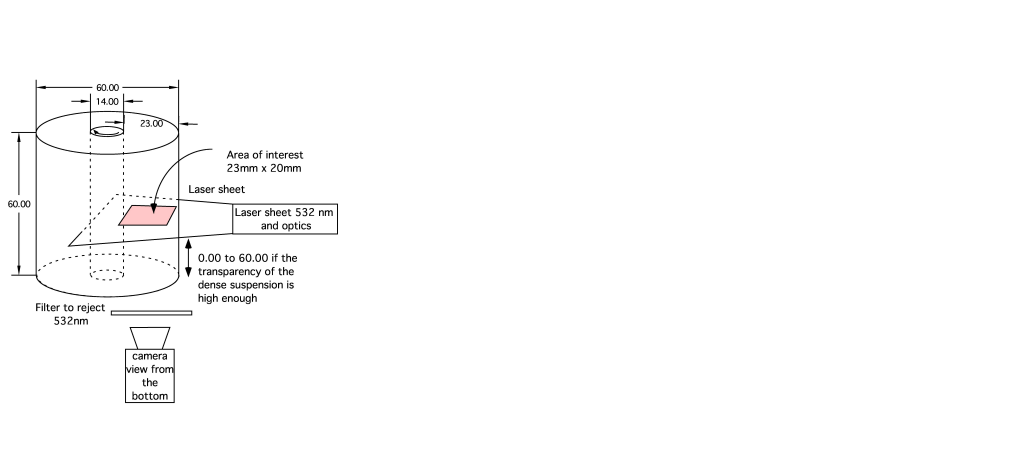


\section{Validation}

\section{Validation measurements}

$$
V_{\theta}(r)=\frac{A}{r}+B r \text { with } A=\frac{R_{\text {in }}^{2} R_{\text {out }}^{2} \Omega}{R_{\text {out }}^{2}-R_{\text {in }}^{2}}, \quad B=\frac{R_{\text {in }}^{2} \Omega}{R_{\text {in }}^{2}-R_{\text {out }}^{2}}
$$
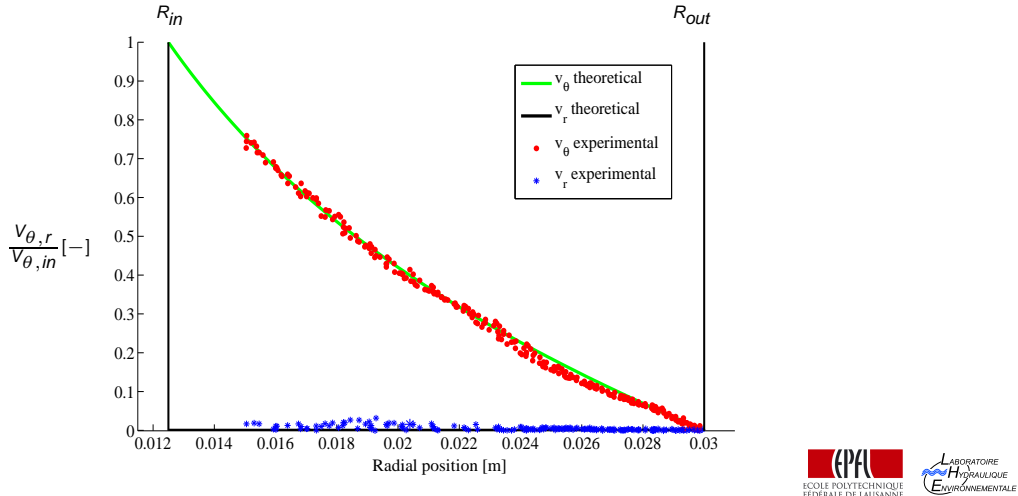

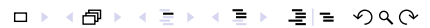




\section{Velocity profile of concentrated suspensions}

\section{Time evolution of the suspension}
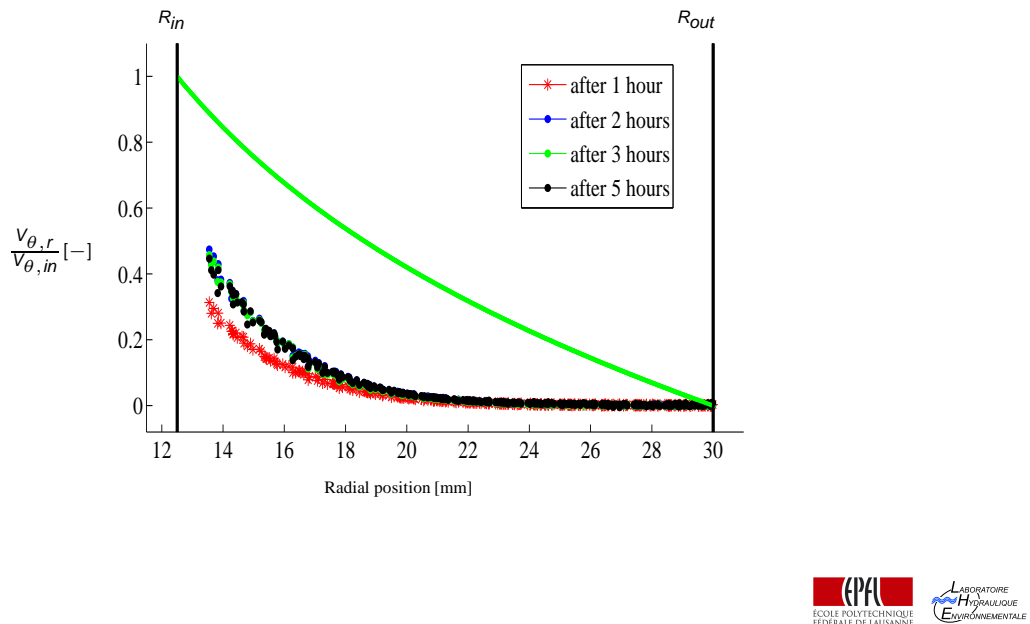

ㅁ 可 


\section{Velocity profile of concentrated suspensions}

\section{Bottom end effects}

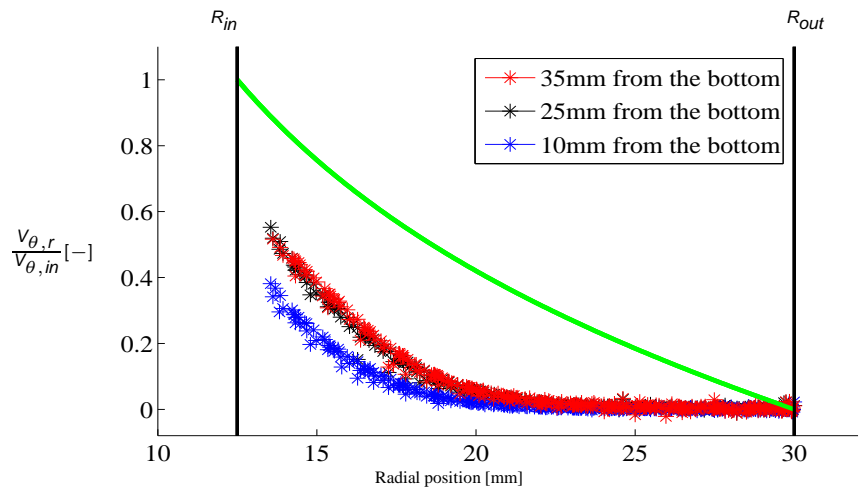

(1)

口 吕 
Flow curve derivation

\section{Flow curve comparison}

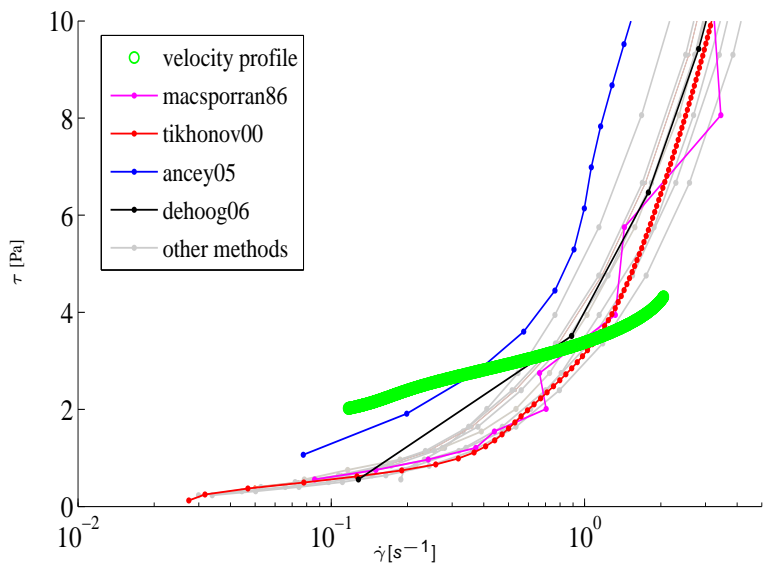

COLE POLYTEChNIOUE

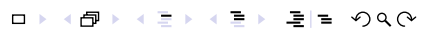




\title{
Flow curve derivation
}

\section{Futur work}

\author{
We want to use the same techniques \\ to make dam-break experiments \\ and measure the inner velocity profile at the front
}

See Poster 165 at the poster session

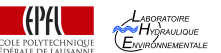

口 向 
Flow curve derivation

\section{Acknowledgment}

- Christophe Ancey

- Nicolas Andreini, Martin Rentschler

- Michel Teuscher and his team

- The Swiss National Science Foundation

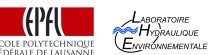


- Iso-index $\Rightarrow$ transparency

- Iso-density $\Rightarrow$ No gravitation effects

- not toxic

Particules

- Sphericity

- Good optical properties

- Granulometry

- Fluorecent molecular tagging
Fluide

- No evaporation

- Wet the PMMA

- Should not disolve PMMA

- Low absorption

- No excitation

- Variable viscosity 


\section{Fluides}

- Lyon (1997)

- Dibromohexane

- Triton X 100

- Huile UCON 75H

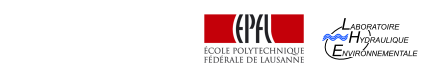

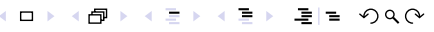




\section{Transparent concentrated noncolloidal suspensions}

- Spherical particles : 200 to $600 \mu \mathrm{m}$

- Iso-index and iso-density fluid mixture

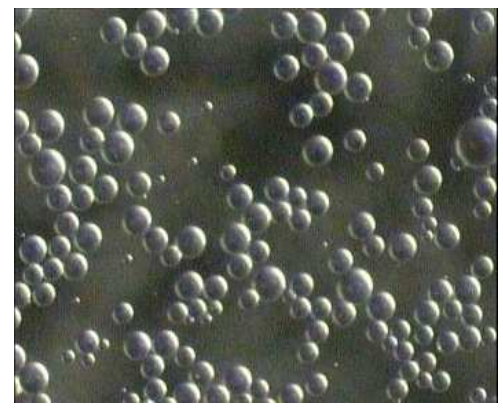

$$
\begin{aligned}
& \text { (1) } \\
& \text { 口 句 }
\end{aligned}
$$




\section{Why Rhodamine 6G ?}

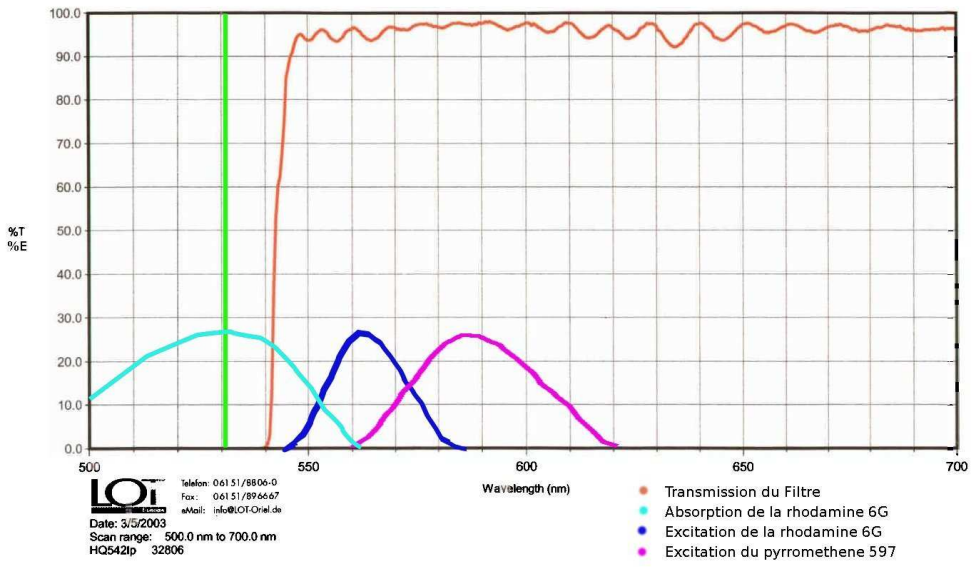

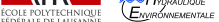

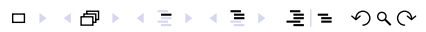




\title{
How much rhodamine 6G?
}

High concentration

\author{
$\Downarrow$ \\ More fluorescence
}

$\Downarrow$

\section{COMPROMIS}

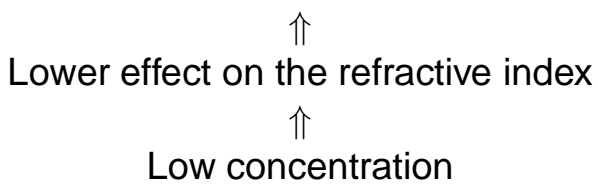

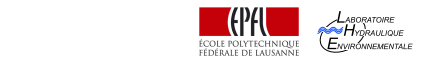

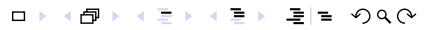




\section{Produit brut}

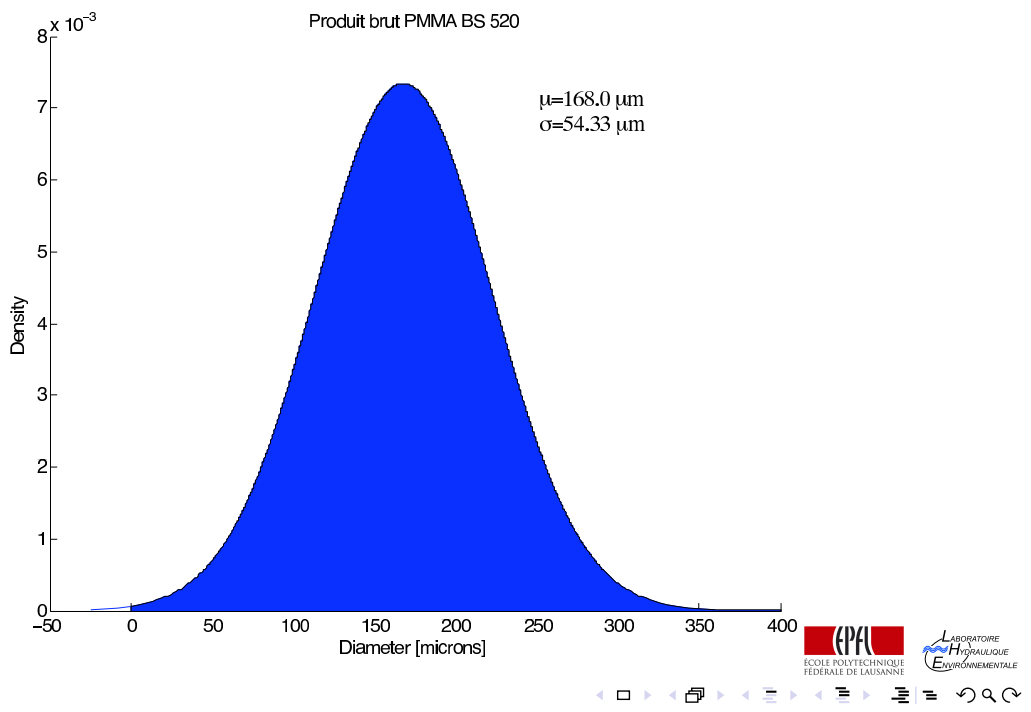




\section{Produit tamisage par voie humide dans de I' ?thanol}

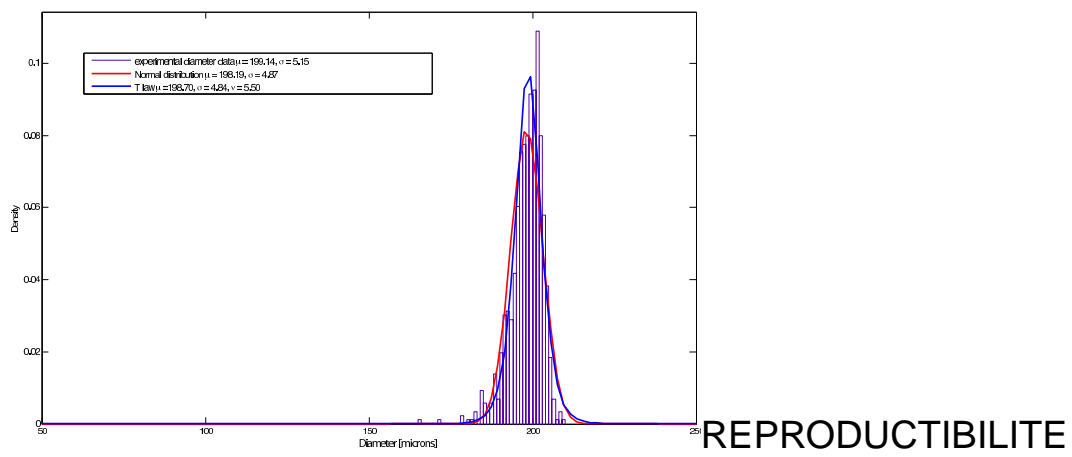

$$
\begin{aligned}
& \text { (fPfID }
\end{aligned}
$$

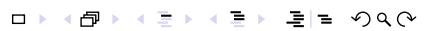




\section{Choix de la Rhodamine 6G}

- Excellent efficacit?

- suffisamment faible "Stokes shift"

Tuning curve Rhodamine 6G

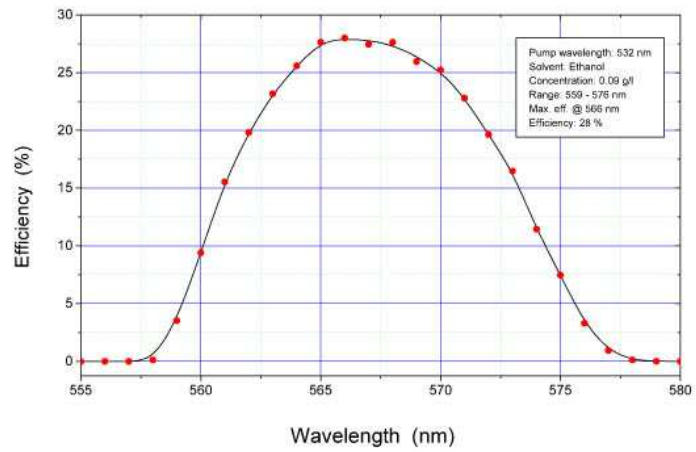

ECOLE POLYTECHNIOUE

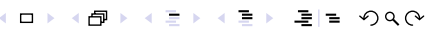




\section{Suspension properties}

- Iso-index $\Rightarrow$ transparency

- Iso-density $\Rightarrow$ No gravitation effects

- Non toxic

Particules

- Sphericity

- Good optical properties

- Granulometry

- Fluorecent molecular tagging
Fluide

- No evaporation

- Wet the PMMA

- Should not disolve PMMA

- Low absorption

- No excitation

- Variable viscosity

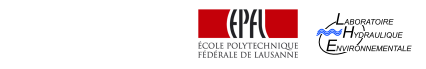

$$
\begin{aligned}
& \text { 口 可 }
\end{aligned}
$$

